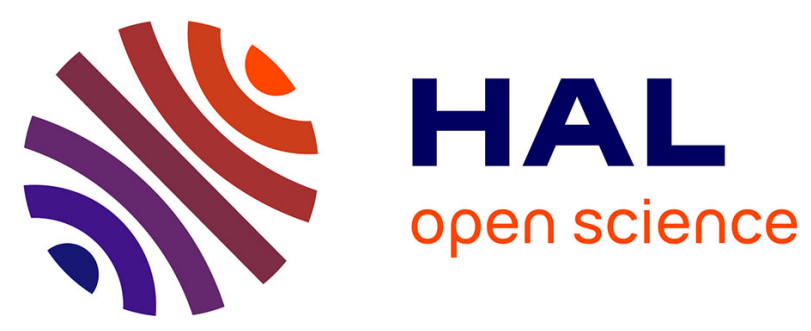

\title{
Toward a standard ontology of surgical process models
}

Bernard Gibaud, Germain Forestier, Carolin Feldmann, Giancarlo Ferrigno, Paulo Gonçalves, Tamás Haidegger, Chantal Julliard, Darko Katić, Hannes Kenngott, Lena Maier-Hein, et al.

\section{- To cite this version:}

Bernard Gibaud, Germain Forestier, Carolin Feldmann, Giancarlo Ferrigno, Paulo Gonçalves, et al.. Toward a standard ontology of surgical process models. International Journal of Computer Assisted Radiology and Surgery, 2018, 13 (9), pp.1397-1408. 10.1007/s11548-018-1824-5 . hal-01862533v3

\section{HAL Id: hal-01862533 https://hal.science/hal-01862533v3}

Submitted on 28 Jun 2021

HAL is a multi-disciplinary open access archive for the deposit and dissemination of scientific research documents, whether they are published or not. The documents may come from teaching and research institutions in France or abroad, or from public or private research centers.
L'archive ouverte pluridisciplinaire HAL, est destinée au dépôt et à la diffusion de documents scientifiques de niveau recherche, publiés ou non, émanant des établissements d'enseignement et de recherche français ou étrangers, des laboratoires publics ou privés. 


\title{
Toward a standard ontology of surgical process models
}

\author{
Bernard Gibaud · Germain Forestier • Carolin Feldmann • Giancarlo \\ Ferrigno · Paulo Gonçalves · Tamás Haidegger • Chantal Julliard · \\ Darko Katić • Hannes Kenngott • Lena Maier-Hein • Keno März • \\ Elena de Momi · Dénes Ákos Nagy • Hirenkumar Nakawala • Juliane \\ Neumann - Thomas Neumuth · Javier Rojas Balderrama - Stefanie \\ Speidel · Martin Wagner · Pierre Jannin
}

\begin{abstract}
Purpose The development of common ontologies has recently been identified as one of the key challenges in the emerging field of surgical data science (SDS). However, past and existing initiatives in the domain of surgery have mainly been focussing on individual groups and failed to achieve widespread international acceptance by the research community. To address this challenge, the authors of this paper launched a European initiative - OntoSPM Collaborative Action - with the goal of establishing a framework for joint development of ontologies in the field of SDS. This manuscript summarizes the goals and the current status of the international initiative.

Methods A workshop was organized in 2016, gathering the main European research groups having experience
\end{abstract}

Bernard Gibaud · Pierre Jannin · Javier Rojas Balderrama Univ Rennes, Inserm, LTSI - UMR_S 1099, Rennes, France

E-mail: bernard.gibaud@univ-rennes1.fr

Carolin Feldmann · Lena Maier-Hein · Keno März

German Cancer Research Center (DKFZ)

Division of Computer Assisted Medical Interventions, Heidelberg, Germany

Giancarlo Ferrigno · Elena de Momi · Hirenkumar Nakawala Politecnico di Milano - NEARLAB, Milan, Italy

Germain Forestier

University of Haute-Alsace - MIPS Laboratory, Mulhouse, France

Paulo Gonçalves

Instituto Politécnico de Castelo Branco, and

IDMEC, Instituto Superior Técnico, Universidade de Lisboa,

Lisboa, Portugal

Tamas Haidegger · Denes Akos Nagy

Óbuda University - Antal Bejczy Center for Intelligent

Robotics, Budapest, Hungary \&

Austrian Center for Medical Innovation and Technology

(ACMIT), Wiener Neustadt, Austria in developing and using ontologies in this domain. It led to the conclusion that a common ontology for Surgical Process Models (SPM) was absolutely needed, and that the existing OntoSPM ontology could provide a good starting point toward the collaborative design and promotion of common, standard ontologies on SPM.

Results The workshop led to the OntoSPM Collaborative Action-launched in mid-2016 - with the objective to develop, maintain and promote the use of common ontologies of SPM relevant to the whole domain of SDS. The fundamental concept, the architecture, the management and curation of the common ontology have been established, making it ready for wider public use. Conclusion The OntoSPM Collaborative Action has been in operation for 24 months, with a growing dedicated membership. Its main result is a modular ontology, undergoing constant updates and extensions, based on the experts' suggestions. It remains an open collabo-

\section{Chantal Julliard}

Université de Montpellier - LIRMM, Montpellier, France, and Univ Rennes, Inserm, LTSI - UMR_S 1099, Rennes, France Present address: Stryker GmbH, Freiburg, Germany

Darko Katić

Karlsruhe Institute of Technology

Institute for Anthropomatics and Robotics, Karlsruhe, Germany

Present address: D. Katić at ArtiMinds Robotics GmbH, Karlsruhe, Germany

Stefanie Speidel

National Center for Tumor Diseases (NCT), Dresden, Germany

Hannes Kenngott · Martin Wagner

University of Heidelberg

Department of General, Abdominal and Transplantation Surgery, Heidelberg, Germany

Juliane Neumann - Thomas Neumuth

Leipzig University - Innovation Center Computer Assisted

Surgery, Leipzig, Germany 
rative action, which always welcomes new contributors and applications.

\section{Introduction}

Context Information processing has become a crucial element of modern surgery, going beyond the guidance of surgical actions using medical images of the patient, rather infiltrating the whole surgical process. Optimization of digital information in the surgical domain is the main objective of an emerging domain, called "Surgical Data Science" (SDS) [42] (Fig. 1).

Information processing is ubiquitous in surgery, deeply impacting all stages of the treatment, from the gathering of preoperative data/images and choosing the most appropriate surgical plan, to the actual realization of the surgery. It may involve robots and other advanced equipment, and advanced statistical tools during the post-operative assessment. In addition to the classical monitoring of vital signs, data-flow often includes the acquisition and interpretation of intra-operative imaging (e.g., video-endoscopy or ultrasound images). The complexity and inter-dependency of these processes require increased automation and optimization in the workflow management. Another challenge that calls for SDS is the consistent communication between all actors and equipment in the Operating Room (OR). Implementing computer systems capable of achieving the previous computing tasks requires to acquire the relevant domain knowledge and to represent it in some machinereadable format. Concretely, this work consists in extensive modeling and analysis of the OR workflows, decomposition of the tasks, identifying the actors involved (both human and robotic) and their roles, in conjunction with the information exchanged [38]. Based on such OR workflow models, context-aware surgical systems aim at interpreting the data provided by sensors (i.e., video cameras, RFIDs on surgical instruments or actors) and apprehending situations in real-time, with respect to predefined models of the procedures, potentially leading to better anticipation of risks, better surgical decision making and most probably, reduction of serious adverse events.

Another application comes from the simulation of such procedural models, contributing to initial and continuing education of healthcare professionals. Training curricula with highly realistic and rich application content can effectively address the needs of training and accreditation of the surgical staff. [14, 37].

Data science offers new possibilities to categorize, learn, and assess from experimental data, annotated in reference to relevant description models and vocabularies. Healthcare management is keen to optimize the use of available resource and equipment, and to prevent adverse events. One of the biggest challenges is to gather enough data, surgical cases from multiple clinics and a variety of patients, in order to overcome the uniqueness of pathologies and procedures. Such pooling requires that multi-center data can actually be semantically aligned, which closely relies on the use of standard protocols, common data models and common vocabularies.

In this context, the ability to create common, standardized Surgical Process Models (SPM), [38] is fundamental and ontologies and semantic technologies are considered to be key enabling technologies for that. Ontologies are information artifacts that present two basic characteristics: they are vocabularies shared in a certain community, and they have formal semantics based on axioms, expressed in some logic language [26]. Description Logics (DL) is a family of widely used knowledge representation languages, especially providing a logical formalism for the Web Ontology language (OWL $)^{1}$. Ontologies can provide significant help to build SPMs, by providing the basic vocabulary (naming and definition of the classes and of the data properties and object properties) that is needed to annotate surgical data. The wider scale adoption of a common vocabulary would greatly facilitate the alignment of data collected in multiple institutions. It would also stimulate the development of specialty-specific procedure models used in workflow management systems and simulationbased training systems. Beyond that, common vocabulary would facilitate the emergence of inter-operability (communication) standards in the OR.

Objective The emergence of SDS and the growing interest for SPMs led to the organization of two complementary workshops in 2016 (Rennes, France and Heidelberg, Germany). The latter (Surgical Data Science workshop) dealt with the strategic organization of the scientific community interested in SDS, and the former (First OntoSPM workshop) dealt with the role of ontologies and semantic technologies in this context. The main European research groups that had been developing ontologies for SPM were invited to share their experience, and to discuss the main challenges and the standardization of their efforts. The following of the paper is organized in three main parts. Section 2 provides an overview of the main contributions reported during the workshop in the domains of SPM and surgical robotics. It reports on the activities which led to launching of the OntoSPM Collaborative Action. Section 3 first describes recent activity of the OntoSPM

1 Web Ontology Language: https://www.w3.org/TR/ owl2-overview/ 


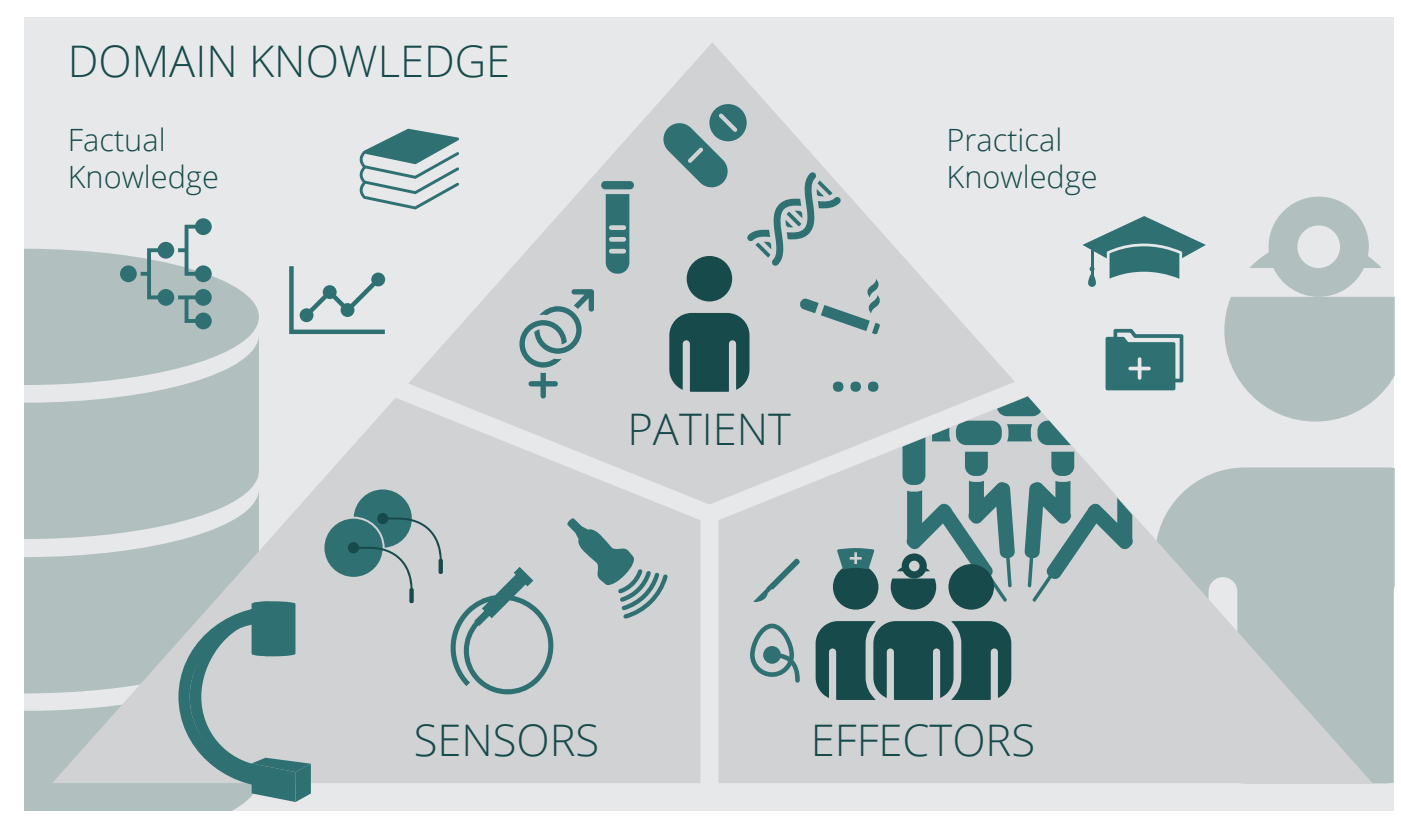

Fig. 1 Illustration of Surgical Data Science. This new domain employs classical data science tools to collect, interpret and optimize data available in the Operating Room to achieve better patient outcome.

Collaborative Action, and it discusses the perspectives and main challenges to face.

\section{The background of SDS research: the results of the First OntoSPM Workshop}

This section provides a summary of the key a priori achievements presented in Rennes, concerning research on SPM and the use of ontologies in medical robotics.

\subsection{Ontology development at ICCAS}

The development of surgical ontologies in the Leipzig University at the Innovation Center for Computer-assisted Surgery (ICCAS) has been associated with the acquisition of intra-operative surgical workflow data using an ontological approach with a surgical workflow editor [55]. The collection of workflow data is used to create verified and valid SPMs [57]. These models are built to support the development of medical devices and computer-assisted systems. In addition, SPMs are a prerequisite for surgical workflow management in modern integrated ORs [13].

The surgical process ontology was implemented for different surgical disciplines and used for the representation of generic surgical processes [55, 59]. Proprietary, discipline-specific ontologies were integrated in a domain-level ontology for surgical workflows [48], and additionally implemented in a Core Ontology for Computer Assisted Surgery (SOCAS) [47]. SOCAS is an on- tological framework, which is embedded into the General Formal Ontology (GFO), and contains various ontologies at different levels of abstraction as well as external ontologies. In addition, an ontology-based meta language was developed, which is a unifying,mathematically founded framework for the modeling of surgical processes [54]. Based on the process ontology, the surgical workflow editor was equipped with an adaptive user interface, which simplifies the recording of workflow data by a sizable terminology for surgical actions, instruments and patient anatomies, considering the actual surgical situation. The ontology-driven interface was validated in a study and enables a recording accuracy above $90 \%$ for inexperienced users [58]. The ontology was also used for the recognition of surgical instruments for the purpose of preselecting the perspectives in the surgical workflow editor [56]. Furthermore, surgical ontologies were developed to support markerless surgical navigation in minimally-invasive endoscopic ENT surgery [3, 73], and for surgical workflow management and automation in the integrated OR [53].

\subsection{Ontology development at the University Hospital of Rennes}

The SDS-related work on at University of Rennes 1 relies on a solid culture and experience of conceptual modeling, acquired along many projects in medical image processing, management and sharing [16, 45, 79]. The first dedicated activities were initiated in the early 2000s, aiming at developing systems facilitating the intra- 
operative management of imaging information. It was applied to neurosurgical procedures (e.g., presenting the right information - target lesion, functional area to be preserved - at the right time and under an appropriate form) [29, 30]. These information models enabled the detailed description of surgical procedures and supported comparison studies (e.g., senior versus novice surgeons $[46,65]$ or practices in different countries [12]). The implementation of such models as formal ontologies (i.e., using ontology languages such as the Web Ontology Language - OWL) was initiated in the context of the French project Synthesis and Simulation of Surgical Process Models (S3PM), aiming to train scrub nurses in neurosurgery using virtual reality [7].

In the context of S3PM, the goal was to provide a formal representation of SPM domain knowledge that is both tailored to the specific needs of this project (i.e. facilitating the creation of simulation scenarios from descriptions of real clinical cases) and also reusable in other application contexts. Based on the S3PM results, two levels were defined in the ontology: a generic one (core ontology), suitable for reuse in almost any domain of surgery and a specific one, focused on the S3PM use cases. The generic module was called OntoSPM as it focused on SPMs, the actions and processes (considered at various granularity levels, from global procedure to detailed actions completed by the actors). The following lines introduce briefly the essential aspects, namely: 1) the domain covered, 2) the relation to an upper level ontology and philosophical framework and 3) the modular architecture with modules extracted from existing ontologies. In terms of scope, key representation included:

- roles played by the actors;

- affected objects (e.g., anatomy or pathology);

- instruments and material used;

- ways of manipulations (e.g., which hand of the surgeon or of the assistant was involved).

A preliminary version of the OntoSPM ontology was presented in 2014 [17], together with a first version of a software based on this ontology, and dedicated to the annotation of surgical procedures from video recordings (SurgeTrack [15]).

This preliminary version of OntoSPM was refined in 2015, in collaboration with the Karlsruhe Institute of Technology (KIT), to make it compatible with the BFO foundational ontology [74], as well as with a number of relevant ontologies that could provide terms (classes and object properties) needed in OntoSPM. This work led to an extended version of OntoSPM, composed of modules extracted from several ontologies developed according to the methodology and principles of the Open Biological and Biomedical Ontologies (OBO) Foundry [75] and reusing the BFO 2 ontology (Table 1).
The extraction of the modules was achieved using the Ontofox tool [83 $]^{2}$ and based on the MIREOT method [8]. Actually, this extended version allowed to meet both initial objectives, i.e. creating realistic simulation scenarios for the S3PM project, and supporting other surgical application contexts, as explained in the next section. The interest raised by OntoSPM in the community led to organization of the First OntoSPM Workshop in Rennes, April 2016. The latter led to the conclusion that the design of an ontology of surgical process models addressing the needs of the whole SDS community required a broader scale collaborative effort. This is the reason why the OntoSPM Collaborative Action was launched in June 2016.

\subsection{Ontology development at the Karlsruhe Institute of Technology}

The development of medical ontologies at KIT, with its partners in Heidelberg and Rennes, focuses on two key areas: surgical workflows in laparoscopy and annotation of surgical data. To support these, two ontologies are developed: the Ontology for Surgical Process Models in Laparoscopy (LapOntoSPM) and the Ontology for Data Integration in Surgery (ODIS). Both are designed as sub-ontologies of OntoSPM. LapOntoSPM inherits OntoSPM's mechanism to model surgical workflows, and extends it with additional concepts for instruments and actions specific to laparoscopic surgeries. Additionally, it models entire surgeries (e.g., laparoscopic adrenalectomies, cholecystectomies and pancreatic resections) at the level of phases and steps. ODIS adds means of describing surgical data and algorithms to OntoSPM.

The primary motivation for the development of LapOntoSPM was the need for a machine-readable representation of surgical knowledge to provide intra-operative context-aware assistance. The focus on this is due to the fact that the availability of intra-operative information outpaces the processing capabilities of surgeons. In the case of sensory overload, information is "physically available" but "not operationally effective" [31]. To counter this, context-aware systems with automatic information filters have been developed. These provide a specific subset, tailored to the given situation in the OR. For this purpose, surgical knowledge is necessary. We represent this-for which the surgical knowledge comes from LapOntoSPM.

LapOntoSPM was used to provide context-aware warnings during cholecystectomies [32], to raise surgeons' attention in critical situations (e.g., when the

2 Ontofox tool: http://ontofox.hegroup.org 
Table 1 Main imported modules of the OntoSPM ontology

\begin{tabular}{|c|c|c|}
\hline FMA & Foundational Model of Anatomy [67] & Domain of human anatomy \\
\hline PATO & Ontology of phenotypic qualities [49] & Domain of phenotypic qualities \\
\hline MPATH & Mouse pathology $[70]$ & $\begin{array}{l}\text { Pathologic entities (for the mouse and } \\
\text { other mammal species) }\end{array}$ \\
\hline $\mathrm{IAO}$ & Information Artifact Ontology [76] & $\begin{array}{l}\text { Information entities in the biomedical } \\
\text { domain }\end{array}$ \\
\hline $\mathrm{UO}$ & Units Ontology [18] & Ontology of units of measurement \\
\hline
\end{tabular}

gallbladder may be harmed). The experiments were conducted on a porcine liver in a realistic environment, as used in training of young surgeons. The outcome was assessed in a structured interview with the performing surgeon. The surgeon confirmed that the surgery is, indeed, facilitated and can be done faster and safer as appropriate warning are given in a timely manner. It was also stressed that the explicit knowledge representation leads to better understanding of the system and makes its behavior more predictable and thus trustworthy. LapOntoSPM enabled these result by allowing us to create straightforward, human-readable rules to define critical situations, and use reasoning to intraoperatively identify them. Furthermore, LapOntoSPM was used to segment entire surgical procedures into phases, employing rule-based reasoning and machine learning [33, 35]. LapOntoSPM successfully aided in this task by enabling the formulation of explicit rules to filter for event indicative of new phases and also helped reduce the number of labeled training samples needed for machine learning. This is especially important in the surgical use case where labeled samples are difficult to acquire. The reliable detection of all relevant phases, as opposed to just critical situations, is an important step towards more sophisticated context-aware assistance during the entire surgery.

ODIS provides a language which allows semantic annotation of surgical data, algorithms and the execution of algorithms on the data [34]. Representation of content types (e.g., image, video, report), actual content (e.g., showing a specific anatomical structure and additionally the patient it belongs to) and provenance (i.e., the origin of data expressed as the processing chain used to obtain it) are all supported by ODIS.

ODIS has been used as the annotation language for the large knowledge base of a semantic data infrastructure, which was created as part of the collaborative research project Cognition Guided Surgery [11]. An early version of ODIS was used in a system to automatically create execution pipelines by combining algorithms for medical use cases [62]. The goal was to automatically sequence processing pipelines for surgical phase recognition based on sensor data and image progressing for tumor progression mappings. ODIS successfully offered the modeling language to describe inputs and outputs of the algorithms so that they could be put in a sensible sequence. ODIS is also part of a system for holistic information processing for surgical decision support in liver surgery [43]. The aim of the project is to support treatment planning for neoplasms based on case knowledge, clinical guidelines and studies. ODIS was successfully used to model the data on which the treatment planning is performed.

\subsection{Ontology development in Heidelberg}

Ontology development in Heidelberg began in 2012, with the Collaborative Research Center 125: "CognitionGuided Surgery", in close collaboration with partners from Karlsruhe (now Dresden) and Rennes. Technical focus has been on the holistic modeling and processing of all relevant data for a given patient with the goal of providing patient-individual clinical decision support [43]. The aim was to provide a model that combines patient data models with factual knowledge in order to allow inferring i.e. prognoses for a patient based on current literature. To this end, two information types are distinguished:

1. Practical knowledge that relates to hospital-specific case data. It represents information that can be extracted from medical images (e.g., tumor number, size and location), laboratory reports or genetic data;

2. Factual knowledge, written down in quotable sources, such as clinical guidelines, studies or educational books.

While most groups in the field have been targeting SPM, Heidelberg has been focusing on to information acquired throughout the whole patient workflow, from diagnosis to therapy and follow-up. First, liver tumor treatment planning was chosen as a clinical application [43], while by now, the ontology comprises over 1000 different parameters extracted from over 200 studies and more than 300 liver tumor patients from the University Hospital Heidelberg. These parameters are linked to the most important biomedical ontologies including SNOMED-CT [82] LOINC [39], RadLex [24] 
and the FMA [67] where possible. The software developed for data annotation and modeling also comprises means for the formalization of studies and clinical guidelines, as well as using them for knowledge inferencing. The model has been applied to several hundreds liver tumor patients, and is currently being extended to applications in renal surgery, including intraoperative process models based on LapOntoSPM. In this context, new methods for large-scale medical data annotation based on crowdsourcing have been developed [25, 40, 41]. An implementation of the system is publicly available [69]. As a first technical use case, the formalization of biomedical image analysis challenges was chosen. The goal was to establish an ontology that:

1. encourages challenge organizers to formalize challenge design in a structured manner to enhance reproducibility and interpretation of results;

2. enables structured access to information from past biomedical image analysis challenges.

The first list comprises a total of more than 50 parameters corresponding to the categories challenge organization, participation conditions, validation objective, study conditions, validation datasets, assessment method and challenge outcome. Using this information, an ontology was created to formalize all biomedical image analysis challenges - about 150 events (challenges) with a total of over 500 competitions (tasks) - that have been conducted until the end of 2016. To apply for a MICCAI challenge 2018, potential organizers needed to submit their proposal in a structured format based on the ontology developed.

\subsection{Ontology development at Politecnico di Milano}

Ontology development in Milan at the Politecnico di Milano started with the ontological description of the robotic components as part of a modular surgical architecture for a robot task execution of a neurological procedure within the ACTIVE (FP7-ICT-2009-6-270460) and the EuRoSurge European projects (FP7-ICT-20117-288233).

A workflow was implemented for the design and the deployment of a modular architecture for autonomous execution of a surgical task (i.e., tool positioning on the correct trajectory for needle insertion), where the architecture's components were automatically derived from the ontological description [61]. The success criteria were defined as final development of modular components which could be safe, need no further re-designing and possibly interchangeable if the surgical scenario is changed. The design of the modular architecture, for surgical autonomous robots, used the knowledge of the robotic components (e.g., connection ports, data types, and priority of sensors) represented in a dedicated ontology module, built on top of the Suggested Upper Merged Ontology (SUMO) [60]. The ontology also contained the device instances available in the lab (e.g., optical trackers). A high-level control was implemented using a component exploiting the incoming trackers' data. The system allowed choosing the best available tracker at each moment, using the ontology, according to the performance of the single sensor to track both the robot and the intra-operative reference frame, and the accuracy of the sensor. The ontology has been shown as a successful design tool for task execution during the robotic surgical workflow.

More recently, an ontology for thoracentesis was developed for context-awareness in surgical training [50]. Thoracentesis is an invasive procedure to remove fluid from the pleural space in disease conditions such as pleural effusion. An ontology named Ontology for Thoracentesis [50] was developed, which was dedicated to context-aware reasoning on the surgical entities and the spatiotemporal activities involved in thoracentesis. To create contextual awareness, the ontology was extended with production rules, which were used to recognize the current phase, as well as instruments on the table and predict the next and previous phases of the surgery. The system was deployed for context-aware surgical training, where the ontology-based system gave the similar results as mentor-based surgical training. Furthermore, production rules were created automatically using the first-order inductive learning [51], which were used in task execution. These contributions can be reused to address the need of integrating entities representing image processing and robotic components within a common ontology of SPM, which could be useful in robotic surgery.

\subsection{Ontology development at the Universidade de Lisboa and the IPCB}

The ontology development for surgical scenarios in Lisbon at Universidade de Lisboa and the Instituto Politécnico de Castelo Branco (IPCB) has been focusing on orthopedics. An Ontology for Robotic Orthopedic Surgery (OROSU) [20] was developed and then applied for hip resurfacing surgery (e.g., for trimming the femoral head). In this scope, the main goal of the research, related to ontologies, was to build a knowledge based framework for this surgical scenario, along with a formal definition of components and actions to be performed during the surgery. The developed ontology was based, in part, on the 1872-2015 - IEEE Standard Ontologies for Robotics and Automation [63]. The 
work was developed under the HIPROB and ECHORD projects, funded by the Portuguese Science Foundation and the EU-FP7, respectively. From the knowledge based framework, several queries can be performed, to extract information from the system, and also for its validation. Amongst others, is possible to monitor the usage of medical devices, and its actions, in the operating room, and to obtain important data from the system sensors, e.g., the current drilling position of the femur.

To evaluate the developed work in a real scenario, a first part of the work was to set up a medical vision system [21] with a robot [81] in the loop, in order to compensate some bone movements when the robot is performing surgical procedures. The second part of the work was to develop the OROSU ontology, based on the Hip Resurfacing scenario. During this study, a survey on ontologies for surgical robotics [19] was performed, concluding that there was still a large room for improvement, to align medical/surgical ontologies to robotic ontologies. This is because of the usage of different upper ontologies, e.g., SUMO and BFO. For the OROSU development, SNOMED CT [82], the CORA ontology [63] and the KnowRob framework [80] were adopted as the base ontologies, and implemented using OWL.

The two outcomes of the research, the robot controlled by a medical vision system, and the OROSU ontology, were amongst the first to integrate robotic ontologies in the surgical field. The system was capable to perform tasks in the hip resurfacing scenario, while interacting with the knowledge base to access, for example, the drilling position of the procedure and the $3 \mathrm{D}$ positions of the medical devices in the operating room. As the conclusions of the studies referenced in this sub-section state, there is still an important work to be done to achieve a full alignment between robotic and surgical/medical ontologies.

\subsection{Ontology development in the IEEE ORA group}

Standardization is becoming an increasingly important area within the overall robotics community, with several works published in the recent years [23], and within IEEE, a special focus has been given to medical/surgical applications, since these lack appropriate regulations and standards [22]. The IEEE 1872-2015 standard [1] refers to the core ontology for Robotics and Automation (R\&A), being the first standard related to ontologies and (non-medical) robotics [68]. "It is composed of a core ontology, called CORA, along with other supporting ontologies, developed under SUMO, thus the basic definitions are derived from SUMO. The supporting ontologies for CORA are: CORAX, RPARTS and POS. The two first cover interaction and robot parts, respectively. The latter (POS ontology) [6] presents the concepts of position, orientation and pose. These concepts are of extreme importance in R\&A. Early adaptation of the CORA includes some medical projects as well.

Currently, the P1872.1 Robot Task Representation Working Group and the P1872.2 Working Group on Autonomous Robotics Ontology are working on application oriented extensions. Nevertheless, these developments remain linked to the field of service robotics.

As a sub-domain of service robotics, surgical robotics is an important cross-section of the medical and R\&A domains. In this domain, clinical-use oriented process models and ontologies have already been proposed for surgical skill evaluation [64], but their implementation in surgical automation and safety standards are still a future work.

\subsection{Discussion}

The convergence of the research topics at many eminent institutions highlighted the relevance and complementarity of the works, and called for synchronized action. These trends allowed to recognize that SPM is a cornerstone of almost all of the projects, thus common, standardized models would be beneficial to the whole community. Bridging the domain of SPM and the domain of medical robotics is important regarding the future development, but still poses many challenges. The OntoSPM ontology was considered as a good starting point, to be further developed and extended, collaboratively. The modality of collaboration was also discussed. The preference was given to an academic collaboration, rather than to a regular standards development process (activities in the context of the DICOM Surgery Working Group could have been an alternative). The reason for this choice was that the topic was felt not enough mature to be well-received by the manufacturers of surgical equipment.

\section{OntoSPM Collaborative Action}

This Section describes the current organization of the OntoSPM Collaborative Action and introduces the main challenges related to its further maintenance and extension towards a broad adoption. 


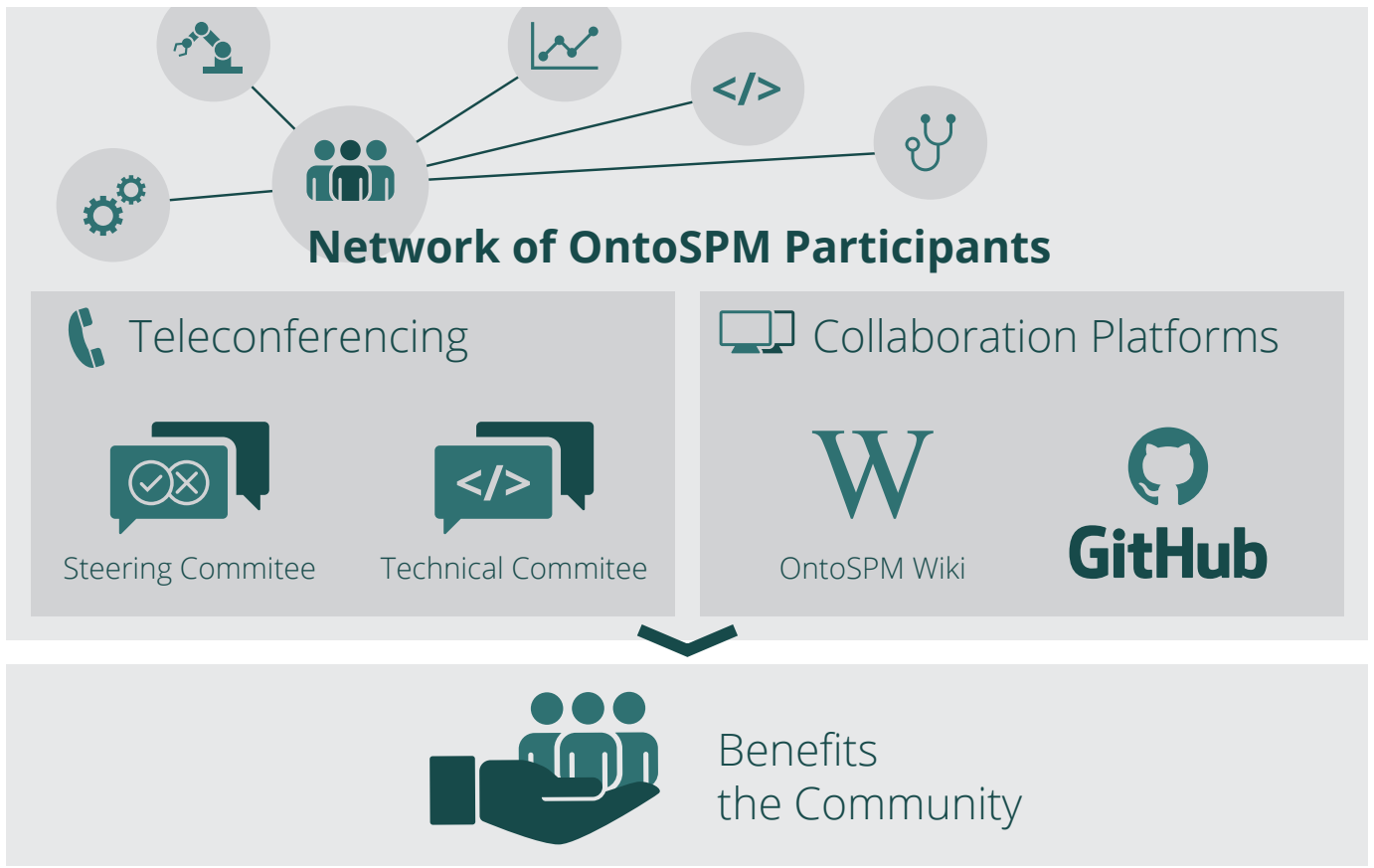

Fig. 2 General organization of the OntoSPM Collaborative Action.

\subsection{Current practical implementation}

The OntoSPM Collaborative Action was launched with the general objective to collaboratively develop and distribute an ontology of Surgical Process Models based on the preliminary work presented above. All the research groups who had contributed to the First OntoSPM workshop were invited to join. The collaboration is organized as a network of participating centers (participants) interacting through teleconferences. A Steering Committee was setup, to coordinate the Action, which includes defining objectives, recruiting new participants, writing proposals or applying for funding, making decisions regarding intellectual property, promoting the Action and its results. The technical work is managed by the Technical Committee, in charge of developing, maintaining and distributing the ontology. New versions of the ontology are delivered regularly and distributed both through a version control repository and through a dedicated website, from which the ontology files can be dereferenced (Fig. 2).

A wiki website ${ }^{3}$ was deployed to support internal and external communication. Internal communication concerns meeting agenda and minutes and document sharing. External communication provides any interested party with general information about the objectives, participants, functioning, and results of the Action. This mode of organization has been in place since

\footnotetext{
3 OntoSPM wiki: https://ontospm.univ-rennes1.fr/ doku.php
}

September 2016. The OntoSPM Collaborative Action currently involves 30 contributors from 15 institutions, located in 7 European countries, and is open to any new contributors.

\subsection{Strategy to extend OntoSPM}

In addition to the need to gather medical experts and improve acceptance of the ontology, the technical development of the ontology itself also faces multiple technical challenges. While the collaborative development of software has been carefully studied and mostly addressed by the software engineering community (source versioning, bug tracking, test-driven development, etc.), such tools are still lacking for ontology engineering. Several ontology development best-practices and methodologies exist $[52,72,77,78]$, but none of them have been accepted at a large scale. As ontology development is in its essence a collaborative work, the lack of mature tools for their collaborative development is a major obstacle. An accepted and important practice in ontology development is the re-use of existing resources to ensure cross-compatibility with existing platforms and acceptance by existing communities. Here again, while the concept of software modules (or software packages) is well-defined and widely used in software development, the best way to implement this concept in ontology engineering is still lacking of maturity [10]. As for the OntoSPM initiative, we decided to re-use as much as possible existing resources through dedicated "ontol- 
ogy modules" containing only an extract of existing resources relevant for our application (e.g., FMA ontology). However, using existing resources also raises the issue of maintaining the created modules according to the evolution of the initial resource, problem known as "dependance maintenance" in software engineering.

For further extending the scope of OntoSPM, the link with existing resources in robotic surgery has also to be strengthened. Discussions are under way in order to link or integrate relevant resources from this domain (e.g., IEEE-CORA). The domain of medical imaging has also to be considered, and existing resources like ODIS would also be relevant to OntoSPM. Some competencies are available within the consortium as it is, but extra participants are welcome, so that to extend ontoSPM into both directions. One of the issues that could prevent or limit the integration of such resources is the choice of different upper-level ontologies on top of these existing resources. Nevertheless, it is certainly feasible to map entities to a different upper ontology; it is fundamentally the role that was assigned to upper level ontologies (as part of the EU WonderWeb Project) to facilitate such mapping [44].

\subsection{Strategy to ensure medical relevance and adoption}

The strategy to ensure medical relevance and acceptance is threefold. First, the development of OntoSPM is directed towards a framework that supports applications for real world surgical use cases and the most pressing challenges in computer-assisted surgery research. Second, practicing surgeons participate in the development of OntoSPM and contribute with their knowledge and feedback. Third, OntoSPM is disseminated into the surgical research community.

Actual clinical application: A critical factor for adoption is that surgeons understand the concrete added value of SPMs in their daily clinical activities. Surgical use cases for OntoSPM so far include monitoring of surgical procedures for context-aware assistance and documentation, support of surgical training and (cognitive) surgical robotics. Applications based on OntoSPM are able to monitor surgical procedures and provide the right assistance in the right situation based on formalized surgical knowledge [32]. After the operation is finished, the surgeon benefits from automatically created reports that summarize the procedure in a standardized way. Thereby, OntoSPM enables comparison of the procedure to other institutions [27]. Furthermore, these reports will augment today's text reports by taking all relevant sensor data and patient information from the procedure into account. Also, applications in surgical training will allow the trainees to benefit from a more standardized feedback. Training will not only be based on sensor information [36] but training results will enable comparison of trainees' performance. Because of its shared vocabulary OntoSPM can help to bridge the gap from the training lab to the OR. Here, applications can monitor the trainee's performance and progress in the OR and subsequently recommend the most appropriate training. Surgical robotics is nowadays dominated by telemanipulators such as the da Vinci Surgical System (Intuitive Surgical Inc.) [66]. In order to overcome their passive role in the OR and become active assistants, surgical robots need to develop an understanding of the surgical procedure, anatomy, actions and potential adverse events. OntoSPM makes this surgical knowledge machine-interpretable, and thus helps the research community to add cognitive capabilities to surgical robots. In this context, the analysis of surgical processes based on data mining and machine learning techniques would also benefit from OntoSPM. For example, [2] faced the problem of surgical data recorded in different international centers that used different surgical vocabularies to record surgical process. In this work, the authors manually aligned the vocabularies between the centers. While this ad-hoc solution can be considered for proofof-concept application, it does not scale up and requires important manual work. This is why providing an international resource allowing to share annotated data and results is now mandatory to reach the next level of datadriven and knowledge-driven applications in surgery.

Actual surgeons' involvement: Participation of surgeons from different countries and surgical specialties in the OntoSPM collaboration is a prerequisite for its success. Yet, it is essential that their important clinical knowledge can be shared with people trained in computer science or knowledge engineering, in order to insure clinical relevance. The organization of summer schools can definitely help creating a common culture shared by surgical research trainees on knowledge engineering and the use of OntoSPM.

Dissemination: For dissemination into the surgical community OntoSPM will be promoted as a framework for standardization in surgical trials. It is a wellknown problem that surgical procedures - in contrast to medication trials - cannot easily be compared because of a lack of standardization [28]. Whereas some trials require their participants to proof their level of competence [5], monitoring the surgical procedure, for example regarding learning curves, requires new approaches [28]. Accordingly standardized reporting of surgical procedures is only in its infancy [4]. Here, the surgeons participating in the collaboration will transfer the results of OntoSPM into their respective surgical communities and describe surgical procedures in pub- 
lications according to the OntoSPM standards. As a long-term goal publications of trial results in high impact journals [2] will not only require a trial registration [9] and adherence to the Consolidated Standards of Reporting Trials (CONSORT) [71], but also standardization of the procedures by means of models and monitoring according to OntoSPM.

\section{Conclusions}

The paper presented the OntoSPM Collaborative Action, which serves as a platform developing ontologies in the domain of surgery, focusing on Surgical Process Modeling in the context of Surgical Data Science. Past efforts and contributions from actual members, related to surgical process models and surgical robotics were presented, as well as the currently active collaboration between several European institutions.

The authors aim at increasing the size and the scope of this international collaborating network, currently limited to the development of the OntoSPM ontology. Current members and their local clinical partners aim at involving the international clinical community to describe needs, discuss knowledge models dedicated to specific domains of surgery and specific procedures. Such discussions and models are supposed to feed the reflection on what OntoSPM should cover in terms of scope in the future.

Based on the experience and expertise of the action partners and the industry needs, we envision that OntoSPM can evolve to cover data science concepts in a ontological framework, to categorize, learn and assess from experimental surgical data. This framework will enable next-generation surgery, delivering better and safer patient care, thanks to the ability to share surgical knowledge and actual surgical data in semanticallyconsistent machine readable form.

\section{Compliance with Ethical Standards}

Conflict of interest The authors declare no conflict of interest.

Human and animal rights This article does not contain any studies with human participants or animal performed by any of the authors.

Informed consent Statement of informed consent was not applicable since the manuscript does not contain any patient data.

Acknowledgements This work was initiated in the context of the S3PM project which received a French government support granted to the CominLabs excellence laboratory and managed by the National Research Agency in the
"Investing for the Future" program under reference ANR-10LABX-07-01. The work on ontological modeling at the DKFZ is supported by the Federal Ministry of Economics and Energy (BMWi) and the German Aerospace Center (DLR). The work on ontological modeling at Politecnico (Milano) has received funding from the European Unions Horizon 2020 research and innovation programme under grant agreement No. H2020-ICT-2016-732515. It was also partly supported by Instituto Politecnico de Castelo Branco and by FCT, through IDMEC, under LAETA, project UID/EMS/50022/2013. T. Haidegger is supported through the New National Excellence Program of the Ministry of Human Capacities, his research was partially supported by the Hungarian OTKA PD 116121 grant. This work has been partially supported by ACMIT (Austrian Center for Medical Innovation and Technology), which is funded within the scope of the COMET (Competence Centers for Excellent Technologies) program of the Austrian Government. We acknowledge the financial support of this work by the Hungarian State and the European Union under the EFOP-3.6.1-16-2016-00010 project. The work at ICCAS was funded by the German Ministry of Education and Research (BMBF).

\section{References}

1. (2015) IEEE standard 1872-2015 - Ontologies for Robotics and Automation, IEEE Robotics and Automation Society. DOI 10.1109/IEEESTD.2015.7084073

2. (2017) Annals of Surgery - Instructions for Authors. http://edmgr.ovid.com/annsurg/accounts/ifauth . htm, accessed December 6th, 2017

3. Bieck R, Heuermann K, Schmidt M, Schmitgen A, Arnold S, Dietz A, Thomas N (2016) Towards an information presentation model of a situation-aware navigation system in functional endoscopic sinus surgery. In: 15. Jahrestagung der Deutschen Gesellschaft für Computerund Roboterassistierte Chirurgie, Bern, Switzerland, CURAC2016

4. Blencowe NS, Mills N, Cook JA, Donovan JL, Rogers CA, Whiting P, Blazeby JM (2016) Standardizing and monitoring the delivery of surgical interventions in randomized clinical trials. British Journal of Surgery 103(10):13771384, DOI 10.1002/bjs.10254

5. Bonjer HJ, Deijen CL, Abis GA, Cuesta MA, van der Pas MH, de Lange-de Klerk ES, Lacy AM, Bemelman WA, Andersson J, Angenete E, Rosenberg J, Fuerst A, Haglind E (2015) A randomized trial of laparoscopic versus open surgery for rectal cancer. New England Journal of Medicine 372(14):1324-1332, DOI 10.1056/ NEJMoa1414882

6. Carbonera JL, Fiorini SR, Prestes E, Jorge VAM, Abel M, Madhavan R, Locoro A, Gonçalves P, Haidegger T, Barreto ME, Schlenoff C (2013) Defining positioning in a core ontology for robotics. In: The IEEE/RSJ International Conference on Intelligent Robots and Systems, Tokyo, Japan, IROS 2013, DOI 10.1109/IROS. 2013.6696603

7. Claude G, Gouranton V, Caillaud B, Gibaud B, Arnaldi B, Jannin P (2016) Synthesis and simulation of surgical process models. In: Medicine Meets Virtual Reality 22, Los Angeles (USA), MMVR22, DOI 10.3233/ 978-1-61499-625-5-63

8. Courtot M, Gibson F, Lister AL, Malone J, Schober D, Brinkman RR, Ruttenberg A (2009) MIREOT: The minimum information to reference an external ontology 
term. In: International Conference on Biomedical Ontology, Buffalo (NY), USA, ICBO 2009, DOI 10.1038/npre. 2009.3576.1

9. De Angelis C, Drazen JM, Frizelle FA, Haug C, Hoey J, Horton R, Kotzin S, Laine C, Marusic A, Overbeke AJP, Schroeder TV, Sox HC, Weyden MBVD (2004) Clinical trial registration: A statement from the international committee of medical journal editors. New England Journal of Medicine 351(12):1250-1251, DOI 10.1056/NEJMe048225

10. Doran P, Tamma V, Iannone L (2007) Ontology module extraction for ontology reuse: An ontology engineering perspective. In: The 16th ACM Conference on Information and Knowledge Management, Lisbon, Portugal, CIKM '07, DOI 10.1145/1321440.1321451

11. Fetzer A, Metzger J, Katić D, März K, Wagner M, Philipp P, Engelhardt S, Weller T, Zelzer S, Franz AM, Schoch N, Heuveline V, Maleshkova M, Rettinger A, Speidel S, Wolf I, Kenngott H, Mehrabi BP Arianeb Müller-Stich, Maier-Hein HP Lena Meinzer, Nolden M (2016) Towards an open-source semantic data infrastructure for integrating clinical and scientific data in cognition-guided surgery. In: SPIE Medical Imaging, San Diego (CA), USA, DOI 10.1117/12.2217163

12. Forestier G, Lalys F, Riffaud L, Collins DL, Meixensberger J, Wassef SN, Neumuth T, Goulet B, Jannin P (2013) Multi-site study of surgical practice in neurosurgery based on surgical process models. Journal of Biomedical Informatics 46(5):822-829, DOI 10.1016/j. jbi.2013.06.006

13. Franke S, Neumuth T (2015) Rule-based medical device adaptation for the digital operating room. In: The 37th International Conference of the IEEE Engineering in Medicine and Biology Society, Milan, Italy, EMBC'15, DOI 10.1109/EMBC.2015.7318712

14. Gallagher AG, O'Sullivan GC (2012) Fundamentals of Surgical Simulation: Principles and Practice. Springer London, DOI 10.1007/978-0-85729-763-1

15. Garraud C, Gibaud B, Penet C, Cazuguel, Dardenne G, Jannin P (2014) An ontology-based software for the analysis of surgical process model. In: Computer-assisted Medical Interventions: Scientific problems, tools and clinical applications, Chambery, France, Surgetica

16. Gibaud B, Garfagni H, Aubry F, Pokropek AT, Chameroy V, Bizais Y, Di Paola R (1988) Standardization in the field of medical image management: The contribution of the MIMOSA model. IEEE Transactions on Medical Imaging 17(1):62-73, DOI 10.1109/42.668695

17. Gibaud B, Penet C, Jannin P (2014) OntoSPM: A core ontology of surgical procedure models. In: Computerassisted Medical Interventions: Scientific problems, tools and clinical applications, Chambery, France, Surgetica

18. Gkoutos GV, Schofield PN, Hoehndorf R (2012) The Units Ontology: A tool for integrating units of measurement in science. Database 2012:1-7, DOI 10.1093/ database/bas033

19. Gonçalves PJS (2016) Ontologies applied to surgical robotics. In: Second Iberian Robotics Conference, Lisbon, Portugal, Robot'2015, DOI 10.1007/978-3-319-27149-1_ 37

20. Gonçalves PJS, Torres PMB (2015) Knowledge representation applied to robotic orthopedic surgery. Robotics and Computer-Integrated Manufacturing 33:90-99, DOI 10.1016/j.rcim.2014.08.014

21. Gonçalves PJS, Torres PMB, Santos F, António R, Catarino N, Martins JMM (2015) A vision system for robotic ultrasound guided orthopaedic surgery. Journal of Intelligent \& Robotic Systems 77(2):327-339, DOI 10.1007/s10846-013-0012-7

22. Haidegger T, S VG (2012) Standardization efforts in medical robotics. In: Modular Surgical Robotics: How can we make it possible?, Saint Paul (MN), USA, Eurosurge

23. Haidegger T, Barreto M, Gonçalves P, Habib MK, Ragavan SKV, Li H, Vaccarella A, Perrone R, Prestes E (2013) Applied ontologies and standards for service robots. Robotics and Autonomous Systems 61(11):12151223, DOI 10.1016/j.robot.2013.05.008

24. Heilbrun ME (2013) Evaluating radlex and real world radiology reporting: are we there yet? J Acad Radio 20(11):1327-1328, DOI 10.1016/j.acra.2013.09.011

25. Heim E, Seitel A, Isensee F, Andrulis J, Stock C, Ross T, Maier-Hein L (2018 (in press)) Clickstream analysis for crowd-based object segmentation with confidence. IEEE Transactions on Pattern Analysis and Machine Intelligence

26. Horrocks I (2008) Ontologies and the Semantic Web. Communications of the ACM 51(12):58-67, DOI 10. 1145/1409360.1409377

27. Huaulmé A, Voros S, Riffaud L, Forestier G, MoreauGaudry A, Jannin P (2017) Distinguishing surgical behavior by sequential pattern discovery. Journal of Biomedical Informatics 67:34-41, DOI 10.1016/j.jbi. 2017.02.001

28. Hüttner F, Doerr-Harim C, Probst P, Tenckhoff S, Knebel P, Diener M (2014) Study methods in evidencebased surgery: Methodological impediments and suggested approaches for the creation and transfer of knowledge in surgery. European Surgical Research 53(1-4):8694, DOI $10.1159 / 000366201$

29. Jannin P, Morandi X (2007) Surgical models for computer-assisted neurosurgery. NeuroImage 37(1):783791, DOI 10.1016/j.neuroimage.2007.05.034

30. Jannin P, Raimbault M, Morandi X, Riffaud L, Gibaud B (2003) Model of surgical procedures for multimodal image-guided neurosurgery. Journal of Computer Aided Surgery 8(2):98-106

31. Joyce JP, Lapinsky GW (1983) A history and overview of the safety parameter display system concept. IEEE Transactions on Nuclear Science 30(1):744-749, DOI 10. 1109/TNS.1983.4332369

32. Katić D, Wekerle AL, Görtler J, Spengler P, Bodenstedt S, Röhl S, Suwelack S, Kenngott HG, Wagner M, MüllerStich BP, Dillmann R, Speidel S (2013) Context-aware augmented reality in laparoscopic surgery. Computerized Medical Imaging and Graphics 37(2):174-182, DOI 10. 1016/j.compmedimag.2013.03.003

33. Katić D, Julliard C, Wekerle AL, Kenngott H, MüllerStich BP, Dillmann R, Speidel S, Jannin B Pierre Gibaud (2015) LapOntoSPM: An ontology for laparoscopic surgeries and its application to surgical phase recognition. International Journal of Computer Assisted Radiology and Surgery 10(9):1427-1434, DOI 10.1007/ s11548-015-1222-1

34. Katić D, Maleshkova M, Engelhardt S, Wolf I, März K, Maier-Hein L, Nolden M, Wagner M, Kenngott H, MllerStich BP, Dillmann R, Speidel S (2016) What does it all mean? Capturing semantics of surgical data and algorithms with ontologies. In: Surgical Data Science Workshop, Heidelberg, Germany

35. Katić D, Schuck J, Wekerle AL, Kenngott H, MüllerStich BP, Dillmann R, Speidel S (2016) Bridging the gap between formal and experience-based knowledge 
for context-aware laparoscopy. International Journal of Computer Assisted Radiology and Surgery 11(6):881888, DOI 10.1007/s11548-016-1379-2

36. Kowalewski KF, Hendrie JD, Schmidt MW, Garrow CR, Bruckner T, Proctor T, Paul S, Adigüzel D, Bodenstedt S, Erben A, Kenngott H, Erben Y, Speidel S, MüllerStich BP, Nickel F (2017) Development and validation of a sensor- and expert model-based training system for laparoscopic surgery: The iSurgeon. Surgical Endoscopy 31(5):2155-2165, DOI 10.1007/s00464-016-5213-2

37. Kowalewski KF, Hendrie JD, Schmidt MW, Proctor T, Paul S, Garrow CR, Kenngott HG, Müller-Stich BP, Nickel F (2017) Validation of the mobile serious game application Touch Surgery for cognitive training and assessment of laparoscopic cholecystectomy. Surgical Endoscopy 31(10):4058-4066, DOI 10.1007/ s00464-017-5452-x

38. Lalys F, Jannin P (2014) Surgical process modelling: A review. International Journal of Computer Assisted Radiology and Surgery 9(3):495-511, DOI 10.1007/ s11548-013-0940-5

39. Lougheed M, Wasilewski N, Morra A, Minard J (2018) Use of SNOMED CT and LOINC to standardize terminology for primary care asthma electronic health records. J Asthma 55(6):629-639, DOI 10.1080/02770903.2017. 1362424

40. Maier-Hein L, Mersmann S, Kondermann D, Bodenstedt S, Sanchez A, Stock C, Kenngott HG, Eisenmann M, Speidel S (2014) Can Masses of Non-Experts Train Highly Accurate Image Classifiers? In: Medical Image Computing and Computer-Assisted Intervention MICCAI 2014, Springer, Cham, Lecture Notes in Computer Science, DOI 10.1007/978-3-319-10470-6_55

41. Maier-Hein L, Ross T, Gröhl J, Glocker B, Bodenstedt S, Stock C, Heim E, Götz M, Wirkert S, Kenngott H, Speidel S, Maier-Hein K (2016) Crowd-Algorithm Collaboration for Large-Scale Endoscopic Image Annotation with Confidence. In: Medical Image Computing and Computer-Assisted Intervention MICCAI 2016, Springer, Cham, Lecture Notes in Computer Science

42. Maier-Hein L, Vedula SS, Speidel S, Navab N, Kikinis R, Park A, Eisenmann M, Feussner H, Forestier G, Giannarou S, Hashizume M, Katic D, Kenngott H, Kranzfelder M, Malpani A, März K, Neumuth T, Padoy N, Pugh C, Schoch N, Stoyanov D, Taylor R, Wagner M, Hager GD, Jannin P (2017) Surgical data science for next-generation interventions. Nature Biomedical Engineering 1(9):691-696, DOI 10.1038/s41551-017-0132-7

43. März K, Hafezi M, Weller T, Saffari A, Nolden M, Fard N, Majlesara A, Zelzer S, Maleshkova M, Volovyk M, Gharabaghi N, Wagner M, Emami G, Engelhardt S, Fetzer A, Kenngott H, Rezai N, Rettinger A, Studer R, Mehrabi A, Maier-Hein L (2015) Toward knowledgebased liver surgery: Holistic information processing for surgical decision support. International Journal of Computer Assisted Radiology and Surgery 10(6):749-759, DOI 10.1007/s11548-015-1187-0

44. Masolo C, Borgo S, Gangemi A, Guarino N, Oltramari A (2003) WonderWeb Deliverable D18. The ontology library. Laboratory For Applied Ontology - ISTC-CNR

45. Mechouche A, Morandi X, Golbreich C, Gibaud B (2009) A hybrid system using symbolic and numeric knowledge for the semantic annotation of sulco-gyral anatomy in brain MRI images. IEEE Transactions on Medical Imaging 28(8):1165-1178, DOI 10.1109/TMI.2009.2026746
46. Morineau T, Morandi X, Le Moëllic N, Diabira S, Riffaud L, Haegelen C, Hénaux PL, Jannin P (2009) Decision making during preoperative surgical planning. Human Factors 51(1):67-77, DOI 10.1177/0018720809332847

47. Mudunuri R, Neumuth T, StraußG, Dietz A, Meixensberger J, Burgert O (2007) SOCAS: Surgical ontologies for computer assisted surgery. In: The 21th International Congress and Exhibition on Computer Assisted Radiology and Surgery, Berlin, Germany, CARS 2007, DOI 10.1007/s11548-007-0114-4

48. Mudunuri R, Burgert O, Neumuth T (2009) Ontological modelling of surgical knowledge. In: Jahrestagung der Gesellschaft für Informatik, Lübeck, Germany

49. Mungall C, Gkoutos G, Washington N, Lewis S (2007) Representing phenotypes in OWL. In: Workshop on OWL: Experiences and Directions, Innsbruck, Austria, OWLED 2007

50. Nakawala H, Ferrigno G, de Momi E (2017) Toward a knowledge-driven context-aware system for surgical assistance. Journal of Medical Robotics Research 2(3):1740,007 1-14, DOI 10.1142/S2424905X17400074

51. Nakawala H, de Momi E, Pescatori LE, Morelli A, Ferrigno $\mathrm{G}$ (2017) Inductive learning of the surgical workflow model through video annotations. In: The IEEE 30th International Symposium on Computer-based Medical Systems, Thessaloniki, Greece, CBMS 2017, DOI 10.1109/CBMS.2017.91

52. Neuhaus F, Vizedom A, Baclawski K, Bennett M, Dean M, Denny M, Grüninger M, Hashemi A, Longstreth T, Obrst L, Ray S, Sriram R, Schneider T, Vegetti M, West M, Yim P (2013) Towards ontology evaluation across the life cycle: The ontology summit 2013. Appl Ontology 8(3):179-194, URL http://dl.acm.org/citation. cfm?id=2594763.2594765

53. Neumann J, Schreiber E, Neumuth T (2016) Ontologybased surgical process modeling by using SNOMED CT concepts and concept model attributes. In: The 30th International Congress and Exhibition on Computer Assisted Radiology and Surgery, Heidelberg, Germany, CARS 2016, DOI 10.1007/s11548-016-1412-5

54. Neumuth D, Loebeb F, Herre H, Neumuth T (2011) Modeling surgical processes: A four-level translational approach. Artificial Intelligence in Medicine 51(3):147-161, DOI 10.1016/j.artmed.2010.12.003

55. Neumuth T, StraußG, Meixensberger J, Lemke HU, Burgert O (2006) Acquisition of process descriptions from surgical interventions. In: International Conference on Database and Expert Systems Applications, Kraków, Poland, DEXA 2006, DOI 10.1007/11827405_59

56. Neumuth T, Czygan M, Goldstein D, StraußG, Meixensberger J, Burgert O (2009) Computer assisted acquisition of surgical process models with a sensor-driven ontology. In: The First workshop on Modeling and Monitoring of Computer Assisted Interventions, London, UK, M2CAI

57. Neumuth T, Jannin P, StraußG, Meixensberger J, Burgert O (2009) Validation of knowledge acquisition for surgical process models. Journal of the American Medical Informatics Association 16(1):72-80, DOI 10.1197/jamia. M2748

58. Neumuth T, Kaschek B, Neumuth D, Ceschia M, Meixensberger J, StraußG, Burgert O (2010) An observation support system with an adaptive ontology-driven user interface for the modeling of complex behaviors during surgical interventions. Behavior Research Methods 42(4):1049-1058, DOI 10.3758/BRM.42.4.1049 
59. Neumuth $\mathrm{T}$, Jannin $\mathrm{P}$, Schlomberg $\mathrm{J}$, Meixensberger J, Wiedemann P, Burgert O (2011) Analysis of surgical intervention populations using generic surgical process models. International Journal of Computer Assisted Radiology and Surgery 6(1):59-72, DOI 10.1007/ s11548-010-0475-y

60. Pease A, Niles I, Li J (2002) The suggested upper merged ontology: A large ontology for the semantic web and its application. In: AAAI-2002 Workshop on Ontologies and the Semantic Web, Edmonton (AB), Canada

61. Perrone R, Nessi F, de Momi E, Boriero F, Capiluppi M, Fiorini P, Ferrigno G (2014) Ontology-based modular architecture for surgical autonomous robots. In: The Hamlyn Symposium on Medical Robotics, London, UK

62. Philipp P, Maleshkova M, Katić D, Weber C, Götz M, Rettinger A, Speidel S, Kämpgen B, Nolden M, Wekerle AL, Dillmann R, Kenngott H, Müller-Stich BP, Studer R (2016) Toward cognitive pipelines of medical assistance algorithms. International Journal of Computer Assisted Radiology and Surgery 11(9):1743-1753, DOI 10.1007/ s11548-015-1322-y

63. Prestes E, Carbonera JL, Rama Fiorini S, M Jorge VA, Abel M, Madhavan R, Locoro A, Gonćalves P, E Barreto M, Habib M, Chibani A, Gérard S, Amirat Y, Schlenoff C (2013) Towards a core ontology for robotics and automation. Robotics and Autonomous Systems 61(11):11931204, DOI 10.1016/j.robot.2013.04.005

64. Reiley CE, Hager GD (2009) Task versus subtask surgical skill evaluation of robotic minimally invasive surgery. In: Medical Image Computing and Computer-Assisted Intervention, London, UK, MICCAI 2009, DOI 10.1007/ 978-3-642-04268-3_54

65. Riffaud L, Neumuth T, Morandi X, Trantakis C, Meixensberger J, Burgert O, Trelhu B, Jannin P (2010) Recording of surgical processes: A study comparing senior and junior neurosurgeons during lumbar disc herniation surgery. Operative Neurosurgery 67(suppl 2):ons325, DOI 10.1227/NEU.0b013e3181f741d7

66. Rodríguez-Sanjuán JC, Gómez-Ruiz M, Trugeda-Carrera S, Manuel-Palazuelos C, López-Useros A, Gómez-Fleitas M (2016) Laparoscopic and robot-assisted laparoscopic digestive surgery: Present and future directions. World Journal of Gastroenterology 22(6):1975-2004, DOI 10. 3748/wjg.v22.i6.1975

67. Rosse C, Mejino Jr JL (2003) A reference ontology for biomedical informatics: The Foundational Model of Anatomy. Journal of Biomedical Informatics 36(6):478500, DOI 10.1016/j.jbi.2003.11.007

68. Schlenoff CI, Prestes E, Madhavan R, Gonçalves P, Li H, Balakirsky SB, Kramer TR, Miguelanez E (2012) An IEEE standard ontology for robotics and automation. In: The IEEE/RSJ International Conference on Intelligent Robots and Systems, Vilamoura, Portugal, IROS 2012, DOI 10.1109/IROS.2012.6385518

69. Schmidt G (2016) Visualizing Knowledge-Based liver tumor treatment planning. PhD thesis, University Koblenz - Landau

70. Schofield PN, Gruenberge M, Sundberg JP (2010) Pathbase and the MPATH ontology: Community resources for mouse histopathology. Veterinary Pathology 47(6):10161020, DOI $10.1177 / 0300985810374845$

71. Schulz KF, Altman DG, Moher D, Fergusson D (2010) CONSORT 2010 changes and testing blindness in RCTs. The Lancet 375(9721):3-9, DOI 10.1016/S0140-6736(10) 60456- 4

72. Schulz S, Jansen L (2013) Formal ontologies in biomedical knowledge representation. Yearbook Medical Informatics 22(1):132-146, DOI 10.1055/s-0038-1638845

73. Siemoleit S, Uciteli A, Bieck R, Herre H (2017) Ontological modelling of situational awareness in surgical interventions. In: Proceedings of the Joint Ontology Workshops (JOWO) 2017, Episode 3: The Tyrolean Autumn of Ontology, CEUR Workshop Proceedings, Vol. 2050, Aachen, 2018, Bolzano, Italy

74. Smith B, Kumar A, Bittner T (2005) Basic formal ontology for bioinformatics. Tech. rep., Institute for Formal Ontology and Medical Information Science, Saarland University, Saarbrücken, Germany

75. Smith B, Ashburner M, Rosse C, Bard J, Bug W, Ceusters W, Goldberg LJ, Eilbeck K, Ireland A, Mungall CJ, The OBI Consortium, Leontis N, Rocca-Serra P, Ruttenberg A, Sansone SA, Scheuermann RH, Shah N, Whetzel PL, Lewis S (2007) The OBO foundry: Coordinated evolution of ontologies to support biomedical data integration. Nature Biotechnology 25:1251-1255, DOI $10.1038 /$ nbt1346

76. Smith B, Ceusters W, Ruttenberg A (2009) From Basic Formal Ontology to the Information Artifact Ontology. In: International Conference on Biomedical Ontology, Buffalo (NY), USA, ICBO 2009

77. Spyns P, Tang Y, Meersman R (2008) An ontology engineering methodology for DOGMA. Applied Ontology 3(1-2):13-39, DOI 10.3233/AO-2008-0047

78. Sure Y, , Angele J, Staab S (2002) OntoEdit: Guiding ontology development by methodology and inferencing. In: On the Move to Meaningful Internet Systems 2002: CoopIS, DOA, and ODBASE, Irvine (CA), USA, OTM 2002, DOI 10.1007/3-540-36124-3_76

79. Temal L, Dojat M, Kassel G, Gibaud B (2008) Towards an ontology for sharing medical images and regions of interest in neuroimaging. Journal of Biomedical Informatics 41(5):766-778, DOI 10.1016/j.jbi.2008.03.002

80. Tenorth M, Beetz M (2013) KnowRob: A knowledge processing infrastructure for cognition-enabled robots. The International Journal of Robotics Research 32(5):566590, DOI $10.1177 / 0278364913481635$

81. Torres PMB, Gonçalves PJS, Martins JMM (2015) Robotic motion compensation for bone movement, using ultrasound images. Industrial Robot: An International Journal 42(5):466-474, DOI 10.1108/IR-12-2014-0435

82. Wang AY, Sable JH, Spackman KA (2002) The SNOMED clinical terms development process: Refinement and analysis of content. In: Proceedings of the AMIA Symposium, San Antonio (TX), USA

83. Xiang Z, Courtot M, Brinkman RR, Ruttenberg A, He Y (2010) Ontofox: Web-based support for ontology reuse. BMC Research Notes 3(1):175, DOI 10.1186/ 1756-0500-3-175 